\title{
AN ASYNCHRONOUS ELECTRIC DRIVE WITH THE INDIRECT CONTROL OF THE OUTPUT VARIABLES
}

\author{
Alexander V. Glazachev ${ }^{1}$, Yuri N. Dementyev ${ }^{1 *}$, Ivan V. Rakov ${ }^{1}$, and Anara D. \\ Umurzakova $^{1,2}$ \\ ${ }^{1}$ Tomsk Polytechnic University, 634050, Tomsk, Russia \\ ${ }^{2}$ Innovative University of Eurasia, 637000, Pavlodar, Kazakhstan
}

\begin{abstract}
The article gives a mathematical description of an asynchronous motor with a device of indirect control of the electromagnetic torque and the angular velocity of the asynchronous motor in the electric drive that allows to prove on its basis the method of monitoring the output variables of the asynchronous electric motor. The simulation model of an asynchronous motor with a device of indirect control of the output variables of the asynchronous motor and the main results of the study have been given.
\end{abstract}

\section{Introduction}

A modern asynchronous electric drive for industrial mechanisms occupies a leading position in all industries. When using modern electric drives, such pump units in the liquid transmission systems [1], there is a constant need to control the output variables of the asynchronous motor (AM), which allows to have the information about the technological output parameters of pump units, to monitor the engines' workload, and maintain the speed within predetermined limits. With the development of semiconductor technology there is a real possibility of regulating the rotation frequency of the AM to provide the required technological parameters of the liquid transmission systems. The use of static frequency converters allows to control the AM of the pumping station in accordance with the specified rate of the technological process of the liquid transmission systems. In addition, knowing the current parameter values and conditions of asynchronous motors enables to provide the monitoring of the technological process of the liquid transmission systems and the behavior of the AM, to control its technical condition, to carry out diagnostics in identifying at the early stages the emerging defects in time to fix them. However, not in all technological processes of the liquid transmission systems it is possible to use pump units regulated by the electric drive with frequency converters because of the high cost and complexity of the applied equipment, performed at full capacity of the AM. Therefore, the most appropriate for the liquid transmission systems is the use of the functional diagram shown in Fig. 1, in which to control the output variables of AM of pump units in normal conditions or in case of failure of the main frequency converter, the indirect control device of the output variables of $\mathrm{AM}$ (DIC) is used.

\footnotetext{
*Corresponding author: dementev@tpu.ru
} 
In the functional scheme of the liquid transmission systems $n$ is logically connected to centrifugal pump units $\left(\mathrm{CP}_{i}\right)$, where $\mathrm{H}_{i}$ - pressure of $i$-th pump unit $(i=1,2, \ldots, n), \mathrm{Q}_{i}-$ feed and flow rate of $i$-th pumping unit $(i=1,2, \ldots, n), L$ - distance.

The most common devices for measuring and controlling the speed are not pulse and tachogenerator sensors, but torque [3, 4] balanced and transmission dynamometers, torsion devices and converter installation. With the help of these devices one may control the output variables of the electric motor in the electric drive, but they have a complex design and require careful installation in conjunction with their rotating parts of the electric drive, which results in low reliability and significantly degrades the performance of the electric drive. In addition, their use may not be possible under the terms of the functioning of the electric drive. In this regard, industrial enterprises need the devices, circuit design and methods that allow to monitor the technological output parameters of the operating units through the output variables of the asynchronous motor, to maintain the speed within predetermined limits in the absence of speed and torque sensors when the necessary information is controlled by indirect methods, for example, the indirect control device.

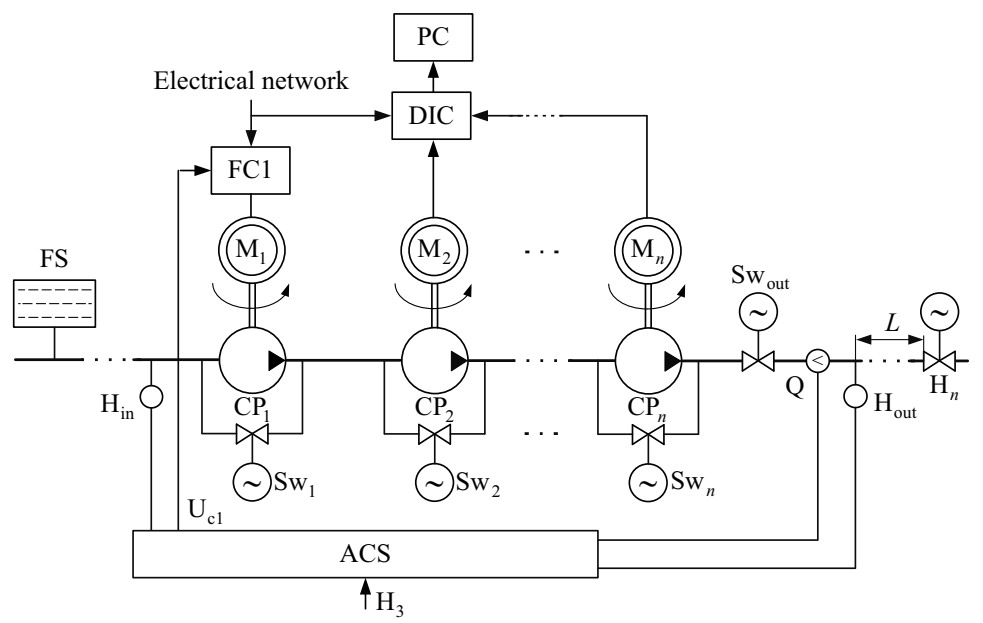

Fig. 1. A Functional Scheme of the Liquid Transmission Systems with One Frequency Converter.

Thus, the development and research of the asynchronous electric drive with the indirect control device of the output variables of the AM is relevant and allows to control the output variables, providing the specified technological parameters.

\section{Mathematical model}

For the mathematical description of the indirect control of the electromagnetic torque and angular velocity, one can use the expressions given in [5].

The mathematical expressions for the indirect determination of the output variables of the asynchronous motor of electromagnetic torque and angular velocity [7-11] can be represented with the following system of equations (1)

$\left\{\begin{array}{l}M(t)=\sqrt{3} p_{n}\left(i_{a}(t) \int\left[u_{b}(t)-R_{s} i_{b}(t)\right] d t-i_{b}(t) \int\left[u_{a}(t)-R_{s} i_{a}(t)\right] d t\right) ; \\ \omega(t)=\omega_{m}(t)\left[1+\Delta \omega_{\mathrm{int}}(t)+\Delta \omega_{\mathrm{dif}}(t)\right],\end{array}\right.$ 
Where $\quad \omega_{m}(t)=\frac{\sqrt{3}\left[u_{a}(t)-\left(R_{s}+R_{r \alpha}^{\prime}\right) i_{a}(t)\right]}{\int_{0}^{1 / f}\left(R_{s}\left[i_{a}(t)+2 i_{b}(t)\right]-\left[u_{a}(t)+2 u_{b}(t)\right]\right) d t-L_{\beta}\left[i_{a}(t)+i_{b}(t)\right]}$

measured the instantaneous value of the angular velocity;

$\Delta \omega_{\text {int }}(t)=\frac{\int\left[u_{a}(t)-R_{s} i_{a}(t)\right] d t}{T_{r}^{\prime}\left[u_{a}(t)-\left(R_{S}+R_{r \alpha}^{\prime}\right) i_{a}(t)\right]}-$ the dynamic integral component of the relative value of the angular velocity; $\Delta \omega_{\mathrm{dif}}(t)=\frac{L_{\beta} \frac{d i_{a}(t)}{d t}}{u_{a}(t)-\left(R_{s}+R_{r \alpha}^{\prime}\right) i_{a}(t)}-$ the dynamic differential component of the relative value of the angular velocity; $R_{r \alpha}^{\prime}=R_{r}^{\prime} \alpha$ - the active reduced resistance of the rotor winding, taking into account $\alpha$ coefficient; $R_{r}^{\prime}$ - the active reduced resistance of the rotor; $\alpha=\frac{L_{s}}{L_{r}^{\prime}}$ - the coefficient equal to the ratio of the total inductance of the stator winding $L_{S}$ to the reduced total inductance of the rotor winding $L_{r}^{\prime}$ ; $L_{\beta}=L_{\mu} \beta-L_{S}-$ the inductance with account of $\beta$ coefficient; $L_{\mu}$ - the mutual inductance of the stator and rotor windings; $\beta=\frac{L_{\mu}}{L_{r}^{\prime}}-$ the coefficient equal to the ratio of the mutual inductance $L_{\mu}$ to the reduced total inductance of the rotor winding $L_{r}^{\prime}$; $T_{r}^{\prime}=\frac{L_{r}^{\prime}}{R_{r}^{\prime}}-$ the rotor time constant.

According to the above equations of the system (1) one can determine the output variables of the asynchronous motor by measuring the voltage, currents of stator phases and data of the electric motor to control their current values.

\section{Mathematical modeling}

On the basis of the abovementioned mathematical description in Fig. 2 in the software package MatLab the simulation model of an asynchronous motor with the indirect control device of the output variables of the asynchronous motor has been made, it allows to carry out studies of the asynchronous electric drive during frequent scalar control excluding PWM of motor supply voltage. 


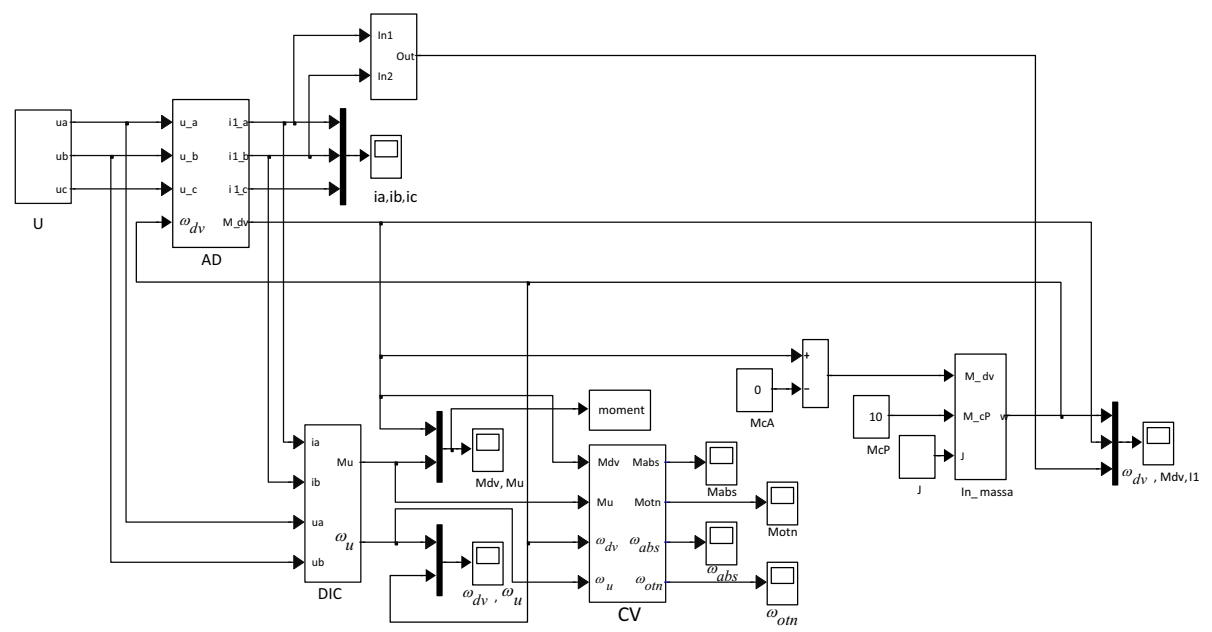

Fig. 2. Simulation model of an asynchronous motor with the indirect control device.

The structure of the simulation model includes superblocks: a model of the asynchronous motor is made based on the mathematical description of the asynchronous motor (AM); phase voltage generator stator windings in a three phase coordinate system (U); inertial mass (In_massa); the indirect control device (DIC) of the electromagnetic torque of the asynchronous motor; a comparator of values obtained of the electromagnetic torque of an asynchronous motor with indirect control devices (CV).

Fig. 3 show the results of simulation studies of the asynchronous motor (AIR90L4) for various values of load, as dependences, respectively, current, speed and torque of time $I(t), M(t), \omega(t)$, which are obtained from an asynchronous motor and indirect control device during frequent scalar control of an asynchronous motor excluding PWM motor supply voltage.
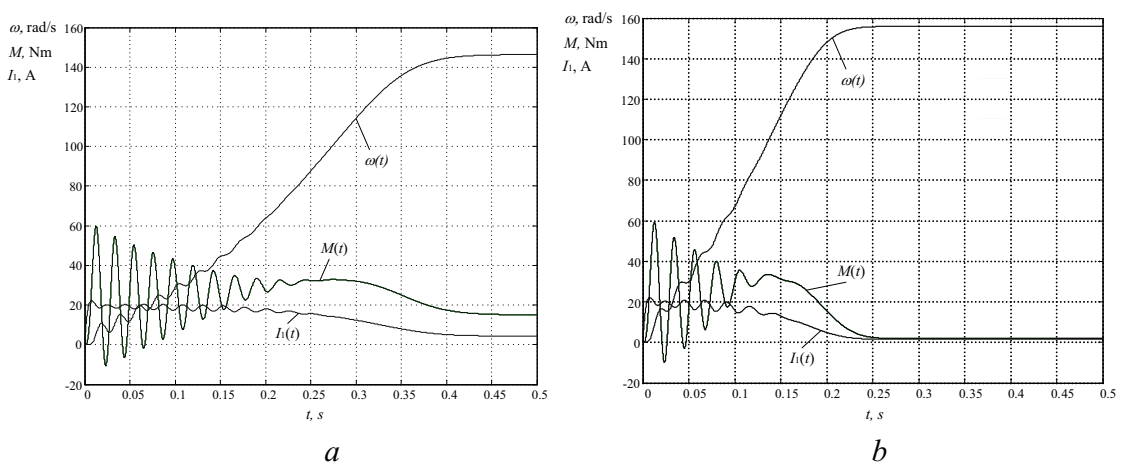


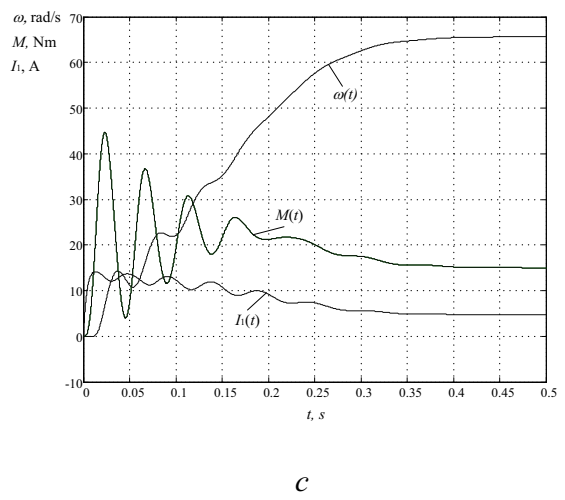

Fig. 3. Dependences $I(t), M(t), \omega(t),, a-f=50 \mathrm{~Hz}, M_{l}=10 \mathrm{Nm} ; b-f=50 \mathrm{~Hz}, M_{l}=2 \mathrm{Nm}$; $c-f=25 \mathrm{~Hz}, M_{l}=15 \mathrm{Nm}$.

From the above-mentioned characteristics, it follows that dependences of the electromagnetic torque and the angular velocity obtained from the output of the asynchronous motor and indirect control device have the convergence permissible in engineering designs.

\section{Results}

To estimate the results of the control of the output variables of the AM in the comparison unit of the simulation model, the absolute and relative inaccuracies of control of the electromagnetic torque and the angular velocity obtained from the AM and the indirect control device are determined, and their values are summarized in Table 1.

Table 1. Absolute and relative inaccuracies of the control of electromagnetic torque and the angular velocity.

\begin{tabular}{|c|c|c|c|}
\hline \multicolumn{2}{|c|}{ Absolute inaccuracy } & \multicolumn{2}{c|}{ Relative inaccuracy, \% } \\
\hline$\Delta M, 10^{-4}, \mathrm{Nm}$ & $\Delta \omega, \mathrm{rad} / \mathrm{s}$ & $\delta_{M}, 10^{-2}$ & $\delta_{\omega}$ \\
\hline$-0,004 \ldots 0,003$ & $-12 \ldots 12$ & $-0,1 \ldots 0.1$ & $-8 \ldots+8$ \\
\hline
\end{tabular}

As seen from the table, the relative inaccuracy of control of the electromagnetic torque is less than $1 \%$, and the angular velocity is not more than $10 \%$ because of the correction link in the speed calculation unit.

\section{Conclusions}

The results of modeling obtained in the study of the asynchronous electric drive with the indirect control device, confirm the correctness of the mathematical description of the asynchronous motor with a device of indirect control of output variables.

According to the studies conducted on the models of the asynchronous motor with the device of indirect control, it has been found that the use of the indirect control device in an asynchronous electric drive allows to conduct the continuous monitoring of electromagnetic torque and angular velocity, and confirms the high efficiency of the indirect control device for electric drives of general-purpose machinery carrying out the technological process and requiring the control of its parameters. 


\section{References}

1. B. Leznov, Energy conservation and a variable drive in pump units (Energoatomizdat, Moscow, 2006) [in Russia]

2. V. Galeev, M. Karkachev, V. Kharlamenko, Main oil pipelines (Nedra, Moscow, 1988) [in Russia]

3. Yu. Rybalchenko, Magnetoelastic torque sensors (Machinostroyenie, Moscow, 1981) [in Russia]

4. M. Leitman, Automatic measuring the output parameters of electric motors (Energoatomizdat, Moscow, 1983) [in Russia]

5. Yu. Dementyev, A. Umurzakova and O. Arsentyev, Bulletin of Irkutsk State Technical University, 7, (2013)

6. Method for measuring the angular velocity of rotation of the three-phase asynchronous motor. (2014, journal, № 23). Patent for invention, Russia № 2525604

7. Yu. Dementyev, A.Umurzakova, K. Khatsevski, Dynamics of systems, mechanisms and machines, 1,(2014)

8. Yu. Dementyev, A. Umurzakova, L. Udut, Fundamental studies, 12, (2014)

9. Yu. Dementyev, A. Umurzakova, MATEC Web of Conf., 19, 010027, (2014)

10. The device for measuring the torque of a three-phase asynchronous electric motor. (2013, journal, № 24). Useful model patent, Russia, № 131874

11. A. Glazachev, Yu. Dementyev, K. Negodin, A. Umursakova, EPJ Web of Conf., 110, 01044, 2016 